\title{
Fabrication of polymer-based reflowed microlenses on optical fibre with control of focal length using differential coating technique
}

\author{
MOHAMMED ASHRAF*, FRANCK CHOLLET, \\ MURUKESHAN MATHAM and CHUN YANG
}

\author{
MicroMachines Centre, School of MAE, Nanyang Technological University, \\ Singapore 639798 \\ e-mail: moha0040@ntu.edu.sg
}

\begin{abstract}
Thermal reflow of polymer to generate spherical profile has been used to fabricate microlenses in this paper. A simple model based on the volume conservation (before and after reflow) and geometrical consideration of lens profile, shows that the focal length of the microlens produced by reflow technique is a function of the initial geometry of microcylinders, i.e. diameter and thickness. This relationship of focal length with diameter and thickness is used as a basis to control focal length. A simple spin coating technique on dual surface is used to achieve differential thickness, to control the focal length of microlenses produced on the same substrate. A biomedical application of such combination of microlenses is endoscopy where the lenses of varying diameter and equal focal length are needed on top of optical fibre bundles to provide independent function of illumination and imaging. This paper incorporates the differential thickness technique to show a micro fabrication process to produce the polymer reflowed microlenses, with a control of focal length based on thickness. The design also helps to integrate these microlenses on top an optical fibre with accurate alignment.
\end{abstract}

Keywords. Microlens; thermal reflow; optical fibre; focal length control; differential coating, polymer optical MEMS.

\section{Introduction}

Thermal reflow of polymer is a simple technique to fabricate refractive microlenses. The technique, which is based on mass transport, was first described by Popovic et al in the year 1988. It uses standard photolithographic process to create photoresist cylinders, which are then reflowed (melted) at an optimum temperature and for an optimum time to transform them into microlens (Popovic et al 1988). During the reflow process, the solid resist is melted, such that the surface tension then pulls these cylinders into a hemispherical shape (Daly et al 1990). If gravitational effects are presumed to be negligible, which for very small lenses will generally be the case, one would expect the shape of these microlenses to be well approximated

*For correspondence 
to a spherical surface (Schilling et al 2000). These hemispherical shapes then act as convex microlenses.

Model based on the volume conservation (before and after reflow) and geometrical consideration of lens profile (approximated to be spherical), indicates that the focal length of the microlens produced by reflow technique is a function of the initial geometry such as diameter and thickness of the micro cylinder. The variation in diameter can be incorporated during the mask design; however, the thickness of the photoresist coated, (which is the thickness of micro cylinder) is usually same throughout the wafer. This limits the scope of application, as there is no way to control the focal length other then varying diameter of the microcylinders. Having a control of microcylinders thickness on to the wafer, without any serious compromise to the process simplicity, would open up new applications (Ashraf et al 2006).

One such application is imaging fibres for endoscopic system as they represent an important constituent of medical endoscopic systems because of their potential application in imaging and sensing. These imaging fibre bundles along with a fibre lens on the tip are used as imaging fibre conduit. However, there is a need for separate illumination and imaging channels, with a control of geometrical aperture and focal length of microlenses. A new generation of imaging fibres with custom made multiple lenses configuration can subdue such limitations by enhancing the illumination areas as well as acceptance cone for imaging. Integration of these lenses on the optical fibre is another issue which limits application.

This paper incorporates the differential thickness technique to show a micro fabrication process on a silicon wafer to produce the polymer-reflowed-microlenses with control of focal length, based on thickness, along with a design to incorporate the packaging of microlenses on top an optical fibre.

\section{Differential coating}

A simple spin coating technique on dual surface is used to achieve differential thickness. The idea is to get a controlled variation in thickness of photoresist on the substrate. This could be achieved by few steps of spin coating. As seen the figure 1, a single coating of resist on a dual surface results in an uneven coating. With multiple coating this unevenness can be reduced. However, a multiple coating may lead to an increase in overall thickness of the photoresist, which may be more than the desired value.

In that case, an RIE-based oxygen plasma etching can evenly remove the excess photoreist to bring down the thickness value to desired number. This way a layer of photoresist with different thickness can be coated on to the single substrate. The dual-surface coating technique can increase the controllability of focal length without actually adding any complex steps to the simple thermal reflow process.

\section{Fabrication details}

Step-wise explanation of the fabrication process to make microlens on top of an optical fibre, incoraporated with differential thickness, is given in figure 2:

1. Base layer of SU8 (negative photoresist) was spin coated on cleaned double sided polished silicon wafer. SU8, being transparent and mechanically strong, provides a good platform to support microlens. The thickness of the layer was varied from $10 \mu \mathrm{m}$ to $50 \mu \mathrm{m}$ based on the subsequent SU8 layer thickness and microlens dimensions. The idea was to keep 


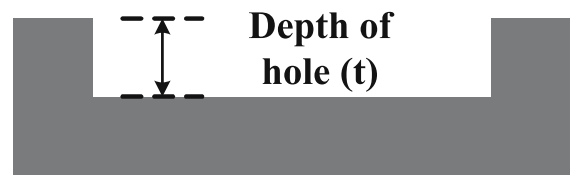

(a) Substrate with a depression/

hole

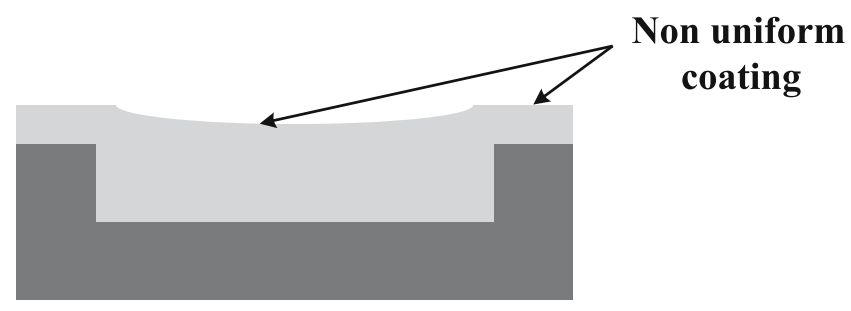

(b) Spin coating of photoresist

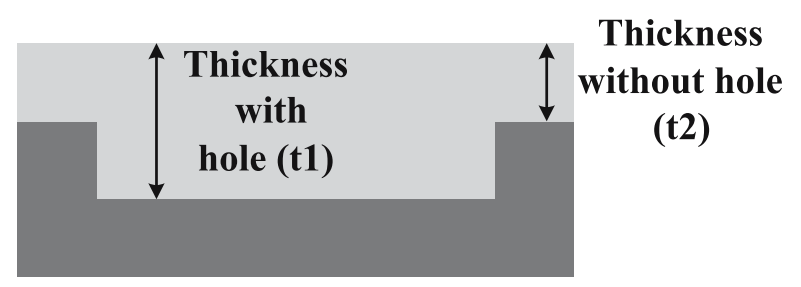

(c) Multiple spin coating to make a uniform layer of photoresist

Figure 1. Multiple spin coating to obtain a uniform layer over a substrate with a depression/hole.

this layer of SU8 as thin as possible, as long as it can sustain the whole lens system. This SU8 layer was then exposed to make it resistant to any chemically harsh environment.

2. A layer $(5 \mu \mathrm{m})$ of AZ9260 was coated on top of SU8 layer, which facilitated the bonding of support wafer.

3. Backside etching of main substrate is done using anisotropic DRIE etching. The support wafer is then carefully removed to avoid any damage to the SU8 layer.

4. A second layer of SU8 layer is coated and patterned to form square holes for dual surface.

5. A layer of AZ9260 is coated over the patterned layer of SU8. This layer acts as the structural material for lens fabrication. Multiple coating is used to achieve a uniform layer.

6. AZ9260 layer is patterned.

7. The patterned micro cylinders are then reflowed at optimum temperature and time to get microlenses.

\section{Results and discussion}

Creating a differential thickness of photoresist on the substrate was tried with four different depths (table 1).

With multiple spin coating this gap was filled, resulting in a uniform surface. As can be seen in figure 3 , the square hole has a depth of $\sim 9 \mu \mathrm{m}$. This difference in depth is reduced to $\sim 6 \mu \mathrm{m}$ with first spin coating (coating 1). 


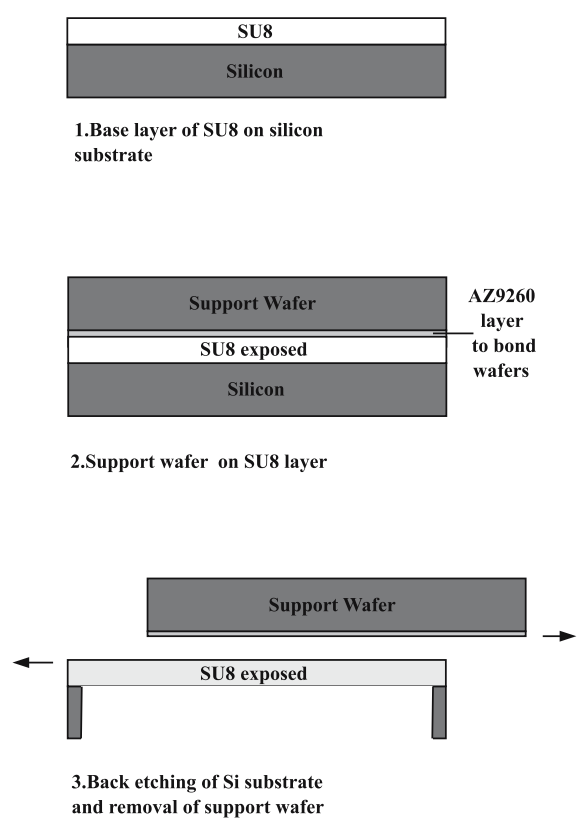

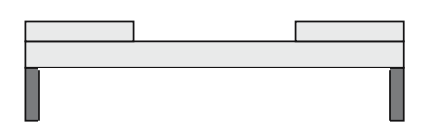

4.Second layer of SU8 patterned

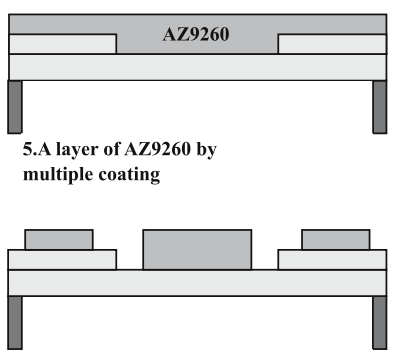

6. AZ layer patterned to make cylinders

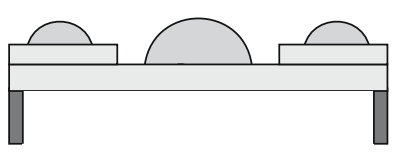

7. PR cylinders melted (reflown)

to form hemispherical lenses

Figure 2. Fabrication steps for microlens on an optical fibre.

However, the coating was not uniform and required another spin coating (coating 2 and 3 ), reducing the difference in height to less than $1.5 \mu \mathrm{m}$. As a result of multiple coating, an increase in the over all thickness of the photoresist may occur, which if, needed, could be reduced by RIE-based oxygen plasma etching.

Table 2 shows the values of parameters used to etch AZ9260. Since the substrate is made of SU8 (negative photoresist), we observed some etching on the SU8 substrate also.

The etching rate for AZ9260 was far greater than that of SU8. However, etching of SU8 is not significant for the process since a thicker layer of SU8 will compensate it.

Table 1. Multiple spin coating to obtain differential thickness (P1-1500 rpm; P2-2000 rpm; P3-3000 rpm; P4-5000 rpm).

\begin{tabular}{lccccc}
\hline $\begin{array}{l}\text { Depth of hole } \\
(t)(\mu \mathrm{m})\end{array}$ & $\begin{array}{c}\text { Differential thickness of } \\
\text { AZ9260 coated }(\mu \mathrm{m}) / \text { spin } \\
\text { coating recipe }\end{array}$ & $\begin{array}{c}\text { Thickness } \\
\text { without hole } \\
(t 2)(\mu \mathrm{m})\end{array}$ & $\begin{array}{c}\text { Thickness } \\
\text { with hole } \\
(t 1)(\mu \mathrm{m})\end{array}$ \\
\hline Sample A & $\mathrm{P} 3$ & $\mathrm{P} 3$ & $\mathrm{P} 4$ & & \\
4.6 & $3 \cdot 9$ & $2 \cdot 4$ & $1 \cdot 4$ & 18 & 23 \\
Sample B & $\mathrm{P} 2$ & $\mathrm{P} 2$ & $\mathrm{P} 4$ & & 36 \\
$9 \cdot 1$ & $6 \cdot 3$ & $2 \cdot 4$ & $1 \cdot 5$ & 27 & 48 \\
Sample C & $\mathrm{P} 2$ & $\mathrm{P} 2$ & $\mathrm{P} 2$ & & 73 \\
$15 \cdot 5$ & $12 \cdot 1$ & $4 \cdot 2$ & $1 \cdot 7$ & 33 & \\
Sample D & $\mathrm{P} 1$ & $\mathrm{P} 1$ & yP1 & & \\
34.6 & 23.9 & $6 \cdot 1$ & 3.5 & 43 & 77 \\
\hline
\end{tabular}




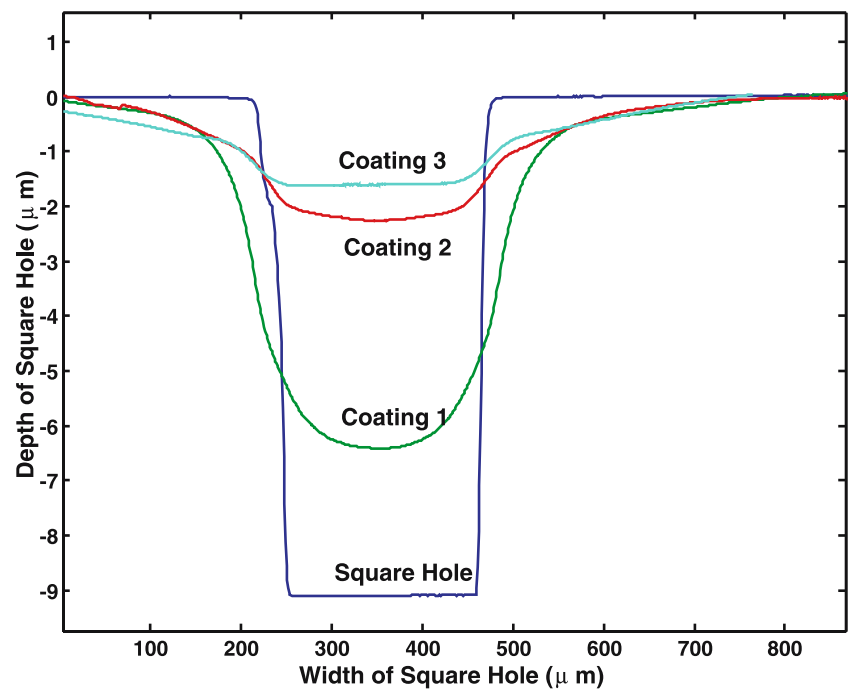

Figure 3. Variation in profile for sample B (measured by stylus profilometer).

Due to different in thickness, which could be significant, exposure and development time of the photoresist have to be optimized for all the samples. A balance was maintained to make sure both the thicker as well as thinner cylinders will develop distinctly (table 3 ).

However, this is not a big issue since all the cylinders are to be reflowed to convert them into microlens, which takes care of any unevenness on the surface of microcylinders.

The micro-cylinders were fabricated with differential thickness, as shown in figure 4 . The overall thickness of the central big cylinder is more than that of outer small cylinders.

Reflow temperature was studied and optimized at $115^{\circ} \mathrm{C}$ for 30 minutes. The process was carried out on a hotplate. Microlens profiles were measured by using the stylus profilometer to estimate the curvature and hence the focal length. Figures 5 and 6 show the microscopic view of lenses on top of SU8 layer, along with the profile.

Table 2. RIE Parameters $\left(0_{2}: 9 \mathrm{sccm}\right.$; $C F_{4}$ : 1 sccm; Power: 200-220 W; Pressure: $88 \mathrm{mTorr})$.

\begin{tabular}{lc}
\hline Time & Thickness (AZ9260) \\
\hline 0 & $23.3 \mu \mathrm{m}$ \\
40 minute & $16.9 \mu \mathrm{m}$ \\
\hline
\end{tabular}

Table 3. Parameters for photolithography.

\begin{tabular}{lcc}
\hline Sample & Exposure time (s) & Development time (s) \\
\hline A & 100 & $180-200$ \\
B & 120 & $200-240$ \\
C & 138 & $240-300$ \\
D & 175 & $420-540$ \\
\hline
\end{tabular}



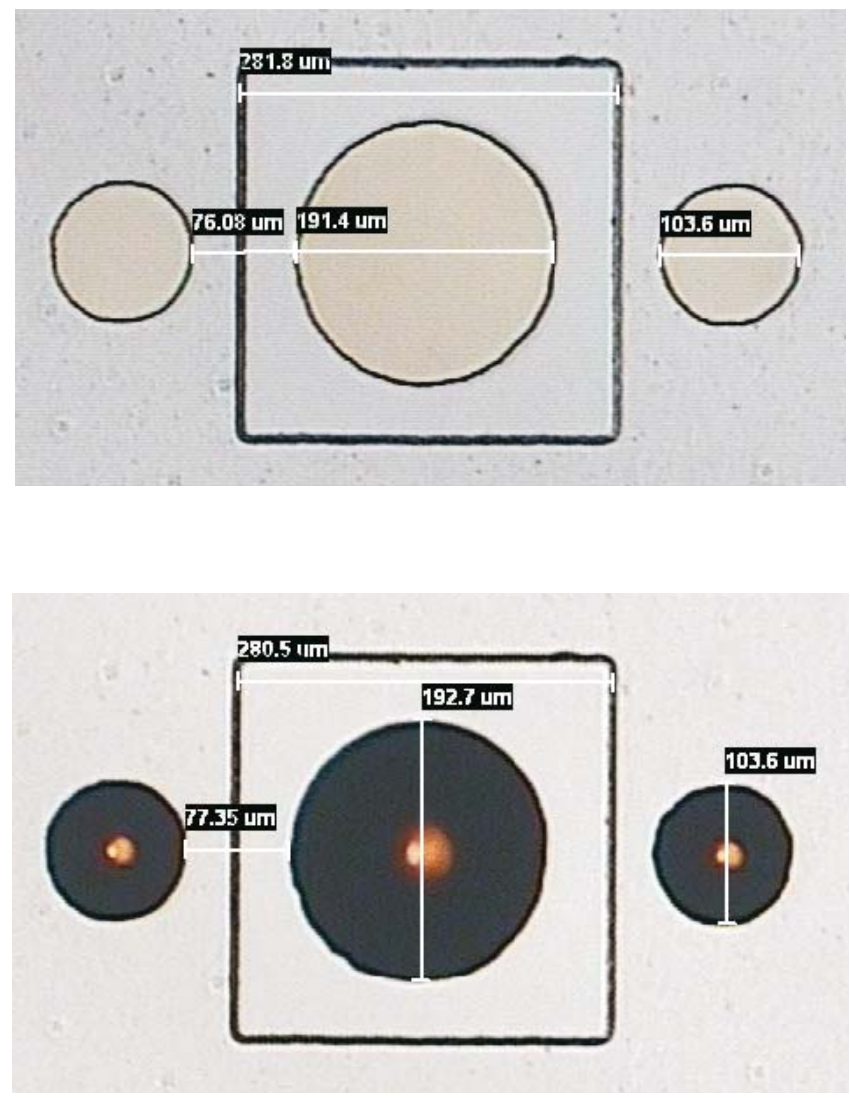

Figure 4. Microscopic view of microcylinders of varying diameter and thickness (before reflow).

Figure 5. Microscopic view of microlens with differential thickness (after reflow).

A square hole could be clearly seen in the microscopic image. A careful inspection of the profile will also reveal the same. Here, the difference in the height of the tip of lenses is not only due to the difference in the original thickness, but also due to the reflow process.

Finally, the optical fibre has been directly inserted into the slot provided by the silicon frame. Thus, it could come in direct contact with the transparent SU8 layer. Figure 7 shows the lenses along with the optical fibre inserted in the slot on the silicon frame.

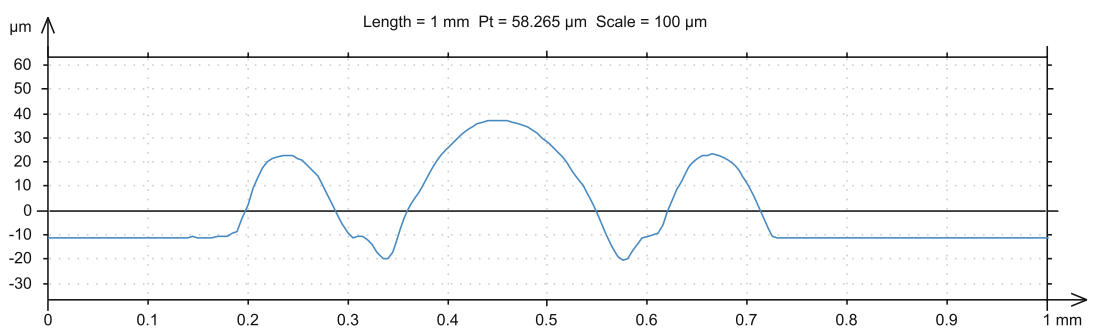

Figure 6. Microlens profile with differential thickness (after reflow). 


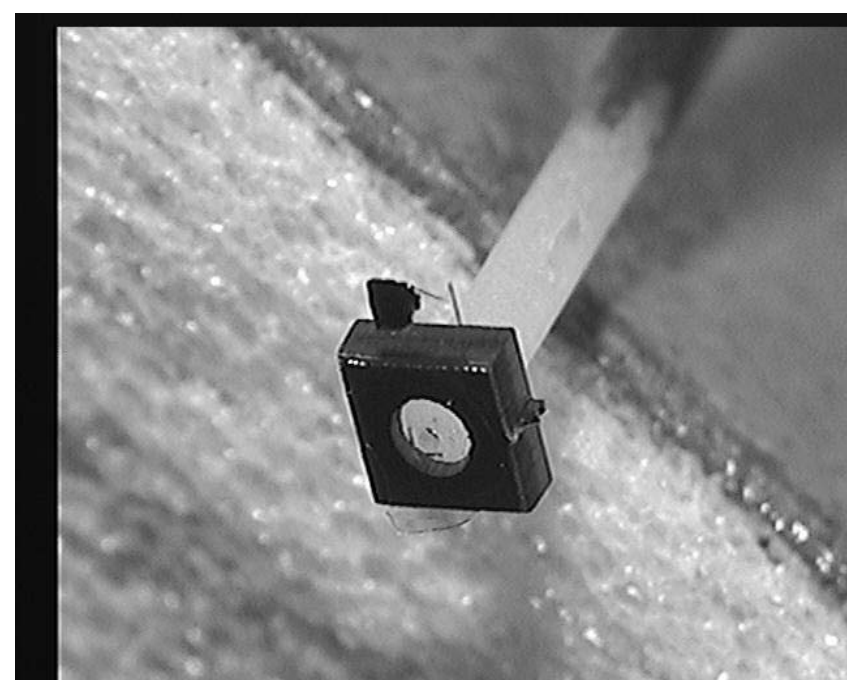

Figure 7. Microlens integrated on optical fibre.

\section{Conclusion}

A simple technique to achieve differential thickness of photoresist on a substrate is shown. The technique has been used to control the profile hence the focal length of the microlens produced by the thermal reflow of photoresist. We have also integrated these microlenses on top of an optical fibre with good alignment.

\section{References}

Ashraf M, Chollet F, Matham M, Yang C 2006 Polymer microlens with independent control of radius and focal length for an imaging fibre. Proceedings of the SPIE 6037: 66-76

Daly D, Stevens R F, Hutley M C, Davies N 1990 The manufacture of microlenses by melting photoresist. Meas. Sci. Technol. 1: 759-766

Popovic Z D, Sprague R A, Connell G A N 1988 Technique for monolithic fabrication of microlens arrays. Appl. Opt. 27: 1281-4

Schilling A, Merz R, Ossmann C, Herzig H P 2000 Surface profiles of reflow microlenses under the influence of surface tension and gravity. Opt. Eng. 39: 2171-2176 